\title{
Neuroimaging Highlight
}

Editors: Mark Hudon, Richard Farb

\section{Herpes Simplex Encephalitis}

\author{
Submitted by: Sean W. Taylor, Roger M. Smith, Giovanna Pari, Wendy Wobeser, \\ John P. Rossiter, Alan C. Jackson
}

Can. J. Neurol. Sci. 2005; 32: 246-247

A 28-year-old woman presented with a one day history of high fever and partial seizures with secondary generalization. This was preceded by a three week history of headache, ataxia, and fatigue. An initial computed tomogram head scan showed a low density mass lesion in the right frontal operculum without enhancement. On the next day, a repeat scan showed a new frontopolar, expansile, low density cortical lesion (Figure 1A) suggestive of encephalitis. Cerebrospinal fluid showed a pleocytosis of 311 mononuclear white blood cell count per $\mu \mathrm{L}$ and an elevated protein of $1.57 \mathrm{~g} / \mathrm{L}$. She received intravenous acyclovir and antibiotics. She remained febrile and became mute. A magnetic resonance (MR) scan under general anesthesia on her fourth hospital day showed frontal and perisylvian lesions with restricted diffusion (Figure 1B - D and Figure 2). A right frontal brain biopsy showed meningoencephalitis and immunohistochemical staining was positive for herpes simplex virus (HSV) antigen (Figure 3). Subsequently, HSV-1 DNA was demonstrated in both cerebrospinal fluid and brain tissue with polymerase chain amplification. She improved after a course of

Figure 1: (A) Contrast enhanced CT head showing low density expansion of cortex and white matter of the right frontal operculum and frontopolar low density cortical lesion with elevation and effacement of the anterior horn of lateral ventricle due to mass effect. There is no enhancement of either cortical lesion. (B) T2 weighted axial image showing perisylvian and frontal cortical distribution of high signal lesions with mass effect effacing the right lateral ventricle. (C) Diffusion weighted image demonstrating curvilinear 'bright' restricted diffusion of the right operculum and frontopolar cortex, which is confirmed by corresponding dark lines (arrowheads) on apparent diffusion coefficient (ADC) mapping (D). Adjacent to the restricted diffusion is the high signal 'T2 shine through' from edema $(D)$.
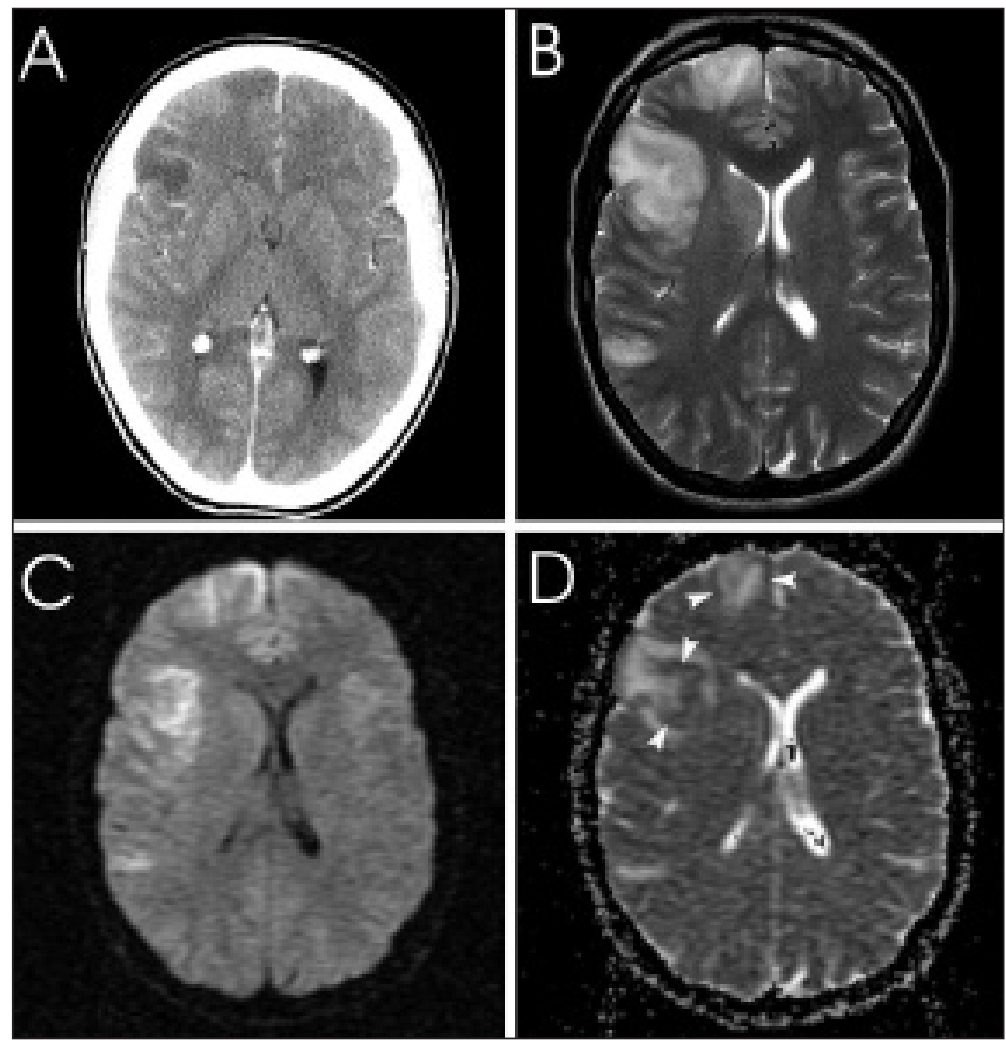

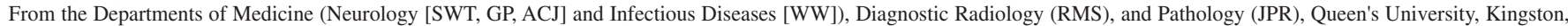
Ontario, Canada

ReCeived August 4, 2004. AcCePted in final form DeCember 6, 2004

Reprint requests to: Alan C. Jackson, Kingston General Hospital, Connell 725, 76 Stuart Street, Kingston, ON K7L 2V7 Canada 


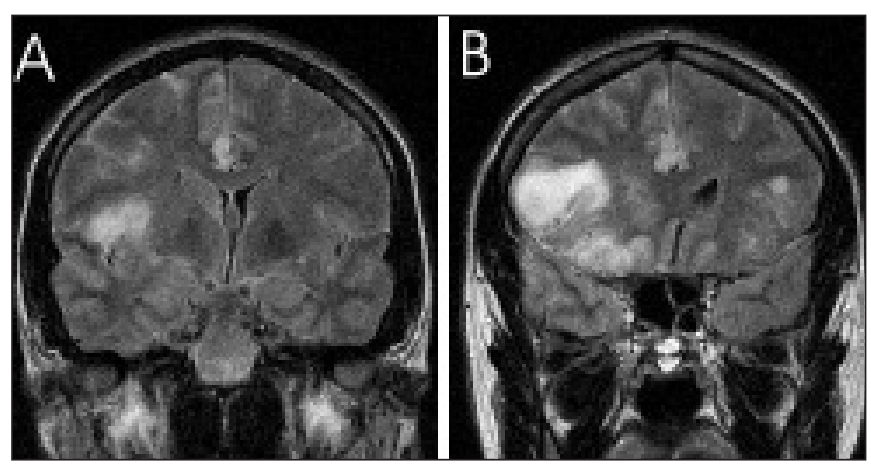

Figure 2: (A and B) Fluid-attenuated inversion recovery coronal images with high signal intensity lesions involving the insula and right cingulate gyrus with sparing of the medial and basal temporal lobe (A) and extensive high signal intensity involvement of the right frontal lobe, cingulate gyrus, and the left frontal lobe $(B)$.

involve medial temporal, insular, orbitofrontal, and cingulate regions. Magnetic resonance imaging is usually abnormal, although rare patients with normal MR studies have been reported. On MR examination, hyperintense signal abnormalities are seen on T2-weighted images at typical sites, including one or both infra-medial temporal lobes, insular cortex, inferior frontal lobes, cingulate gyrus, and thalamus. Occasionally there are also signal characteristics of hemorrhage that persist due to the presence of hemosiderin. ${ }^{2,3}$ The affected areas are hypointense on T1-weighted images. Meningeal enhancement may be demonstrated following administration of

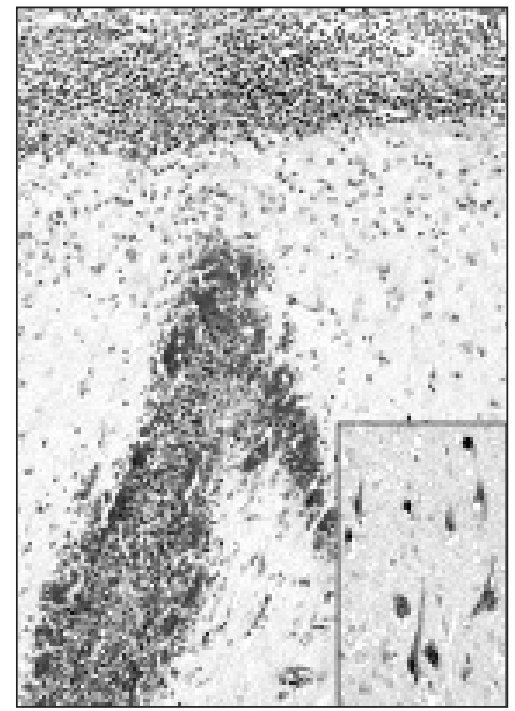

Figure 3: Photomicrograph showing dense mononuclear inflammatory cell infiltrates and microhemorrhages in the leptomeninges (top) and surrounding a penetrating blood vessel in the cerebral cortex (hematoxylinphloxine-saffron stain X 70). Inset: Immunoperoxidase staining showing HSV antigen in the nuclei, cytoplasm, and processes of cortical neurons $X 105$. gadolinium. ${ }^{2}$ Fluid-attenuated inversion recovery sequences demonstrate superior definition of temporal lobe abnormalities compared to standard T1- and T2-weighted images. ${ }^{4}$ Diffusion MR studies may also be useful for early detection of lesions. ${ }^{5}$ Rarely, patients may have lesions in unusual locations, including the parietal and occipital lobes ${ }^{6,7}$ and cerebellum. ${ }^{8}$ Unusual clinical presentations, including the opercular syndrome, ${ }^{9}$ may be difficult to recognize as HSE. The present patient lacked the typical medial temporal lobe involvement often seen in HSE and exhibited prominent frontal lobe lesions.

The frontotemporal localization of HSV in adults is thought to relate to the route of viral entry of HSV into the brain. In 1979, Davis and Johnson ${ }^{10}$ hypothesized that reactivated HSV, which is often latent in trigeminal ganglia, may spread along the trigeminal nerve fibers in tentorial nerves that innervate the basal meninges of the anterior and middle fossae. It has also been postulated that HSV may enter the brain along an olfactory pathway and spread along the base of the brain, particularly during primary HSV infection. The precise factors responsible for maintaining the frontotemporal localization of the infection are uncertain, although the immune response likely plays an important role. Atypical features may occur in immunodeficient patients, ${ }^{11,12}$ while neonates and young children often have a diffuse encephalitis or multifocal lesions. ${ }^{3}$

Unfortunately, the morbidity of HSE remains high despite antiviral therapy. Early initiation of therapy is important. Hopefully, the future will bring new and more effective therapies, including antiviral and neuroprotective agents, for the management of patients with HSE.

\section{REFERENCES}

1. Whitley RJ, Alford CA, Hirsch MS, et al., NIAID Collaborative Antiviral Study Group. Vidarabine versus acyclovir therapy in herpes simplex encephalitis. N Engl J Med 1986;314:144-149.

2. Demaerel P, Wilms G, Robberecht W, et al. MRI of herpes simplex encephalitis. Neuroradiology 1992;34:490-493.

3. Tien RD, Felsberg GJ, Osumi AK. Herpes virus infections of the CNS: MR findings. Am J Roentgenol 1993;1:167-176.

4. White ML, Edwards-Brown MK. Fluid attenuated inversion recovery (FLAIR) MRI of herpes encephalitis. J Comput Assist Tomogr 1995;19:501-502.

5. McCabe K, Tyler K, Tanabe J. Diffusion-weighted MRI abnormalities as a clue to the diagnosis of herpes simplex encephalitis. Neurology 2003;61:1015-1016.

6. Ohta K, Tabata M, Nakachi I, Obara K. HSV encephalitis with posterior localization. Neurology 2003;61:862.

7. Bergey GK, Coyle PK, Krumholz A, Niedermeyer E. Herpes simplex encephalitis with occipital localization. Arch Neurol 1982;39:312-313.

8. Ohta K, Funaki M, Tanaka M, Suzuki N. Early cerebellar involvement on diffusion-weighted magnetic resonance images in herpes simplex encephalitis (Letter). J Neurol 1999;246:736738.

9. McGrath NM, Anderson NE, Hope JK, Croxson MC, Powell KF. Anterior opercular syndrome, caused by herpes simplex encephalitis. Neurology 1997;49:494-497.

10. Davis LE, Johnson RT. An explanation for the localization of herpes simplex encephalitis. Ann Neurol 1979;5:2-5.

11. Chretien F, Belec L, Hilton DA, et al. Herpes simplex virus type 1 encephalitis in acquired immunodeficiency syndrome. Neuropathol Appl Neurobiol 1996;22:394-404.

12. Schiff D, Rosenblum MK. Herpes simplex encephalitis (HSE) and the immunocompromised: a clinical and autopsy study of HSE in the settings of cancer and human immunodeficiency virus-type 1 infection. Hum Pathol 1998;29:215-222. 